\title{
A Quantum Culture Shift
}

\author{
To maintain its leadership in quantum computing technology, the US \\ must embrace a culture shift that fuses three important elements: \\ science, a roadmap, and agility.
}

By Darío Gil, Joseph S. Broz, and Irfan Siddiqi

This piece is the first in a three-part series. Find out more about this series in this introductory article, Ingredients for a Quantum Future.

The promise of quantum technology is real. By storing and manipulating information in quantum bits, or qubits, quantum computers have the potential to solve problems that are far beyond the reach of today's computers. Quantum machines could simulate the properties of the natural world in ways that would transform the design and development of pharmaceuticals (including future vaccines) or of new energy-sustainability materials, such as solar cells and longer lasting batteries. The machines could speed up artificial intelligence and data science tasks, such as the dynamic selection of investment portfolios. And they could allow us to probe the mysteries of the physical Universe. In short, quantum

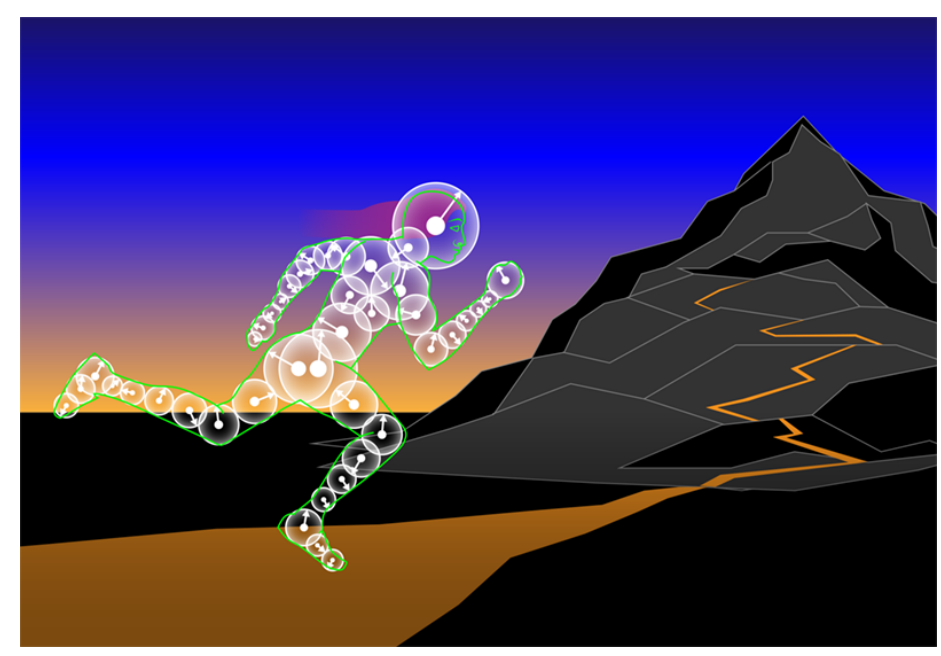

Credit: APS/Alan Stonebraker computing could solve the unsolvable.

The US currently leads in quantum computing R\&D. Its quantum infrastructure is immense, and it has the expertise and technological prowess to further advance the quantum computing frontier. But to maintain and ensure a leadership position, the US needs to sharpen its focus, accelerate the quantum innovation cycle, and boost commercialization. Currently, the country's expertise is distributed among universities, labs, and companies. We must better align these resources to avoid any deceleration in progress, remove unnecessary duplication, and prevent inefficient use of precious national resources. And we must transform our mindset. That is why the three of us are calling on leaders in the government-industry-academia ecosystem to plan for and embrace a "quantum culture shift" that will leverage the nation's full science and technology (S\&T) enterprise.

In our view, this shift should splice together three powerful cultures. The scientific culture, which seeks to always get the fundamentals right. The roadmap culture, which adopts the expertise and development mindset from the world of product manufacturing and systems. And the agile culture, which-as exemplified by the software world-continuously demonstrates value. An agile culture evolves in response to technical advances and user feedback, as opposed to the prevalent "waterfall" approach to innovation, where one development phase is completed before moving on to the next.

Let's start with the scientific culture. At its core, innovative research drives a long-term outlook through a foundation in solid science. Universities have always been a wellspring of new ideas, and they can serve as proving grounds for high-risk, high-reward explorations into quantum fundamentals. In 
tandem, the national labs have the expertise, facilities, and federal-funding commitment to perform exploratory and long-term research. This step is needed to transform new ideas into prototypes and to perform large-scale testing and characterization. Finally, industry, through its corporate research divisions, sits in a critical position between science and product applications. By working in concert with the academic and national labs, industry can create economic opportunity for promising innovations.

Building on the scientific culture, the roadmap culture defines and uses consistent metrics to drive steady progress that compounds on itself. The best-known example is the roadmap that led to Moore's Law. For decades, the International Technology Roadmap for Semiconductors (ITRS) coordinated trillions of dollars of cumulative investments across hundreds of independent organizations doing semiconductor R\&D. This coordination allowed teams and organizations to build essential interdependencies. Consider lithography, the method needed to sculpt tiny circuits onto silicon chips. Developing this essential tool required coordination between materials suppliers and the manufacturers of semiconductor equipment.

In a similar vein, the nascent quantum industry needs a roadmap if it wants to "solve the unsolvable." A roadmap would define meaningful outcomes that go beyond qubit number to include, for instance, error rates, gate connectivity, and parallelism. A good example in this direction is "quantum volume," a recently introduced metric for how well a given system performs as a quantum computer. A roadmap would also assess the value of near-term hardware for building increasingly complex quantum circuits, and it would establish time and risk windows for the introduction of novel ideas, innovations, and enabling technologies.

Lastly, the agile culture is historically associated with manufacturing and software development. It is rooted in speed, user-driven innovation, and feedback. Agility underlies the way that today's most promising quantum software is being advanced-through open source codes and community development. But more importantly, an agile methodology can transcend the traditional path of tech development, which starts with small-scale research teams, who then try to convince larger organizations to develop their work and manufacture a product. Instead of this serial relay race, academia, research labs, national labs, and industry must try, and fail, and learn fast together by efficiently collaborating on parallel cycles of design and user-driven experimentation. The emergence of cloud-enabled quantum computers, which allows hundreds of thousands of users to learn, experiment, and collaborate, shows that this type of parallel effort is possible.

Cultural historians tell us that the pathway to change generally proceeds in three phases: discovery, reward, and diffusion. A new approach is adopted only after society sees that it offers a better outcome. Cultures are also often slow to mutate because they get used to doing things a certain way and because change is uncomfortable when it introduces risk that did not previously exist. Leaders in the quantum community need to offset this cultural inertia. They must provide a clear vision for accomplishing change. Establishing this vision is essential for the US economy and national security, and it will lead to the breakthrough discoveries of tomorrow and a sustained competitive edge in quantum technology.

The next article in this series, Quantum for All, will discuss the need for greater awareness and literacy about quantum concepts outside the quantum community.

Darío Gil: IBM Research, Yorktown Heights, NY, USA

Joseph S. Broz: Quantum Economic Development Consortium (QED-C), Arlington, VA, USA

Irfan Siddiqi: University of California, Berkeley, and Lawrence Berkeley National Laboratory, CA, USA 